\title{
BMO ESTIMATES AND RADIAL GROWTH OF BLOCH FUNCTIONS
}

\author{
BY B. KORENBLUM ${ }^{1}$
}

1. Introduction. A real harmonic function $u(z)$ in the open unit disk $\mathbf{D}$ belongs to the (real) Bloch space $B$ if

$$
\|u\|_{B}=\sup \left\{\left(1-|z|^{2}\right)|\operatorname{grad} u|: z \in \mathbf{D}\right\}<\infty .
$$

The importance of the class $B$ in both complex and harmonic analysis is highlighted by the following facts:

(a) If $f(z)$ is a univalent analytic function in $\mathbf{D}$, then $u(z)=\log \left|f^{\prime}(z)\right| \in B$ and $\|u\|_{B} \leq 8$; conversely, if $u(z) \in B$ and $\|u\|_{B} \leq 1$, then there is a univalent analytic function $f(z)$ in $D$ such that $u(z)=\log \left|f^{\prime}(z)\right|$ (see $[5$, pp. 32, 172]).

(b) The functions $u \in B$ coincide (up to a constant) with the derivatives $(\partial / \partial x) U\left(r e^{i x}\right)$ of harmonic functions $U(z)$ such that $U\left(e^{i x}\right)$ belongs to the Zygmund class $\Lambda_{*}$ (see [6, Chapter $\left.7, \S 3\right]$ ). $0)$ :

Integrating (1) yields the following "trivial" estimate (we assume $u(0)=$

$$
|u(z)| \leq \frac{1}{2}\|u\|_{B} \log \frac{1+|z|}{1-|z|} \quad(z \in \mathbf{D}) .
$$

However, for $L_{p}$ norms of $u(z)$ on circles $|z|=r(0<r<1)$ a much deeper estimate, due to J. G. Clunie and T. H. MacGregor [1] , holds $(p>0)$ :

$$
\|u(r, \cdot)\|_{p}=\left(\frac{1}{2 \pi} \int_{0}^{2 \pi}\left|u\left(r e^{i x}\right)\right|^{p} d x\right)^{1 / p} \leq A_{p}\|u\|_{B} \sqrt{|\log (1-r)|},
$$

where $A_{p}$ are some constants (the case $p=2$ was proved earlier by T. M. Flett $[\mathbf{2}$, p. 71]). As shown in [1], (3) implies that

$$
\lim _{r \rightarrow 1^{-}} \frac{u\left(r e^{i x}\right)}{|\log (1-r)|^{\gamma}}=0
$$

for almost all $x$ whenever $\gamma>1 / 2$.

The need to better understand the nature of such "nontrivial" estimates as (3) and (4) is the main motivation of this paper. In this context the use of the BMO norm for estimating Bloch functions on the circles $|z|=r$ looks more promising, since both norms are invariant under Möbius shifts of the argument.

In what follows, a simple but sharp BMO estimate for Bloch functions is proved. This estimate, together with the John-Nirenberg theorem [3], is then used to obtain a stronger form of the asymptotic estimate (4).

The author expresses his deep gratitude to T. H. MacGregor and R. O'Neil for many stimulating discussions.

Received by the editors June 25, 1984 and, in revised form, July 7, 1984 .

1980 Mathematics Subject Classification. Primary 30C55, 42 A99.

${ }^{1}$ Supported by NSF grant MCS 82-01460. 


\section{BMO estimate.}

DEFINITION 1. $B$ is the real Banach space of (real) harmonic functions $u(z)$ in $\mathbf{D}$ modulo constants with norm (1).

DEFINITION 2. BMO is the real Banach space of (real) $2 \pi$-periodic functions $f(x) \in L^{2}(0,2 \pi)$ modulo constants with norm

$$
\|f\|_{\mathrm{BMO}}^{2}=\sup \left\{U_{f^{2}}(z)-\left[U_{f}(z)\right]^{2}: z \in \mathbf{D}\right\}<\infty,
$$

where $U_{f}(z)$ is the Poisson integral of $f(x)$. It is easily seen that

$$
U_{f^{2}}(z)-\left[U_{f}(z)\right]^{2}=\frac{1}{2 \pi} \int_{0}^{2 \pi}\left[f(t)-U_{f}(z)\right]^{2} P(t, z) d t
$$

where $P(t, z)$ is the Poisson kernel

$$
P(t, z)=\frac{1-|z|^{2}}{\left|e^{i t}-z\right|^{2}}=\frac{\partial}{\partial n_{\zeta}} \log \left|\frac{\zeta-z}{1-\bar{z} \zeta}\right|
$$

$\left(n_{\zeta}\right.$ is the outward normal to $\partial \mathbf{D}$ at $\zeta=e^{i t}$ ).

REMARK. The norm (5) is sometimes called the Garsia norm; it is equivalent to the original Nirenberg BMO norm (see, e.g., [4, p. 329]).

THEOREM 1. Let $u \in B$ and $f_{r}(x)=u\left(r e^{i x}\right)$. Then

$$
\left\|f_{r}\right\|_{\mathrm{BMO}} \leq 2^{-1 / 2}\|u\|_{B} \sqrt{\left|\log \left(1-r^{2}\right)\right|} \quad(0<r<1) .
$$

Proof. By Green's formula $\left(\zeta=e^{\imath t}\right)$,

$$
\begin{aligned}
U_{f_{r}^{2}}(z)-\left[U_{f_{r}}(z)\right]^{2} & =\frac{1}{2 \pi} \int_{0}^{2 \pi}\left[u\left(r e^{i t}\right)-u(r z)\right]^{2} \frac{\partial}{\partial n_{\zeta}} \log \left|\frac{\varsigma-z}{1-\bar{z}_{\zeta}}\right| d t \\
& =\frac{r^{2}}{\pi} \iint_{D}-\left.\log \left|\frac{w-z}{1-\bar{z} w}\right| \operatorname{lgrad} u(r w)\right|^{2} d A_{w} \\
& \leq \frac{r^{2}\|u\|_{B}^{2}}{\pi} \iint_{D}-\log \left|\frac{w-z}{1-\bar{z} w}\right|\left(1-r^{2}|w|^{2}\right)^{-2} d A_{w}
\end{aligned}
$$

where $d A_{w}$ is the Euclidean area element. The latter integral represents a superharmonic function $V(z)$ in $\mathbf{D}$ such that $V(z)=V(|z|)$ and $V\left(1^{-}\right)=0$. Therefore, $\max \{V(z): z \in \mathbf{D}\}=V(0)$ and

$$
\begin{aligned}
\left\|f_{r}\right\|_{\mathrm{BMO}}^{2} & \leq \frac{r^{2}\|u\|_{B}^{2}}{\pi} \iint_{D} \log \frac{1}{|w|}\left(1-r^{2}|w|^{2}\right)^{-2} d A_{w} \\
& =2 r^{2}\|u\|_{B}^{2} \int_{0}^{1}|\log \rho|\left(1-r^{2} \rho^{2}\right)^{-2} \rho d \rho \\
& =\frac{1}{2}\|u\|_{B}^{2}\left|\log \left(1-r^{2}\right)\right|,
\end{aligned}
$$

which completes the proof.

REMARK. The example $u\left(r e^{i x}\right)=r \operatorname{Cos} x$ shows that the constant $2^{-1 / 2}$ in $(7)$ is sharp. The example

$$
u\left(r e^{i x}\right)=\sum_{n=1}^{\infty} r^{k^{n}} \operatorname{Cos}\left(k^{n} x\right)
$$

where $k \geq 2$, shows that $\sqrt{\left|\log \left(1-r^{2}\right)\right|}$ cannot be replaced by a function of slower growth (cf. [1] ). 


\section{Exponential estimate.}

THEOREM 2. There are positive numerical constants $c$ and $C$ such that for all $u(z) \in B, u(0)=0$,

$$
\int_{0}^{2 \pi} \exp \left\{\frac{c\left|u\left(r e^{i x}\right)\right|}{\|u\|_{B} \sqrt{\left|\log \left(1-r^{2}\right)\right|}}\right\} d x \leq C \quad(0<r<1) .
$$

ProOF. By the John-Nirenberg theorem [3] the distribution function $m(y)$ of an $f \in$ BMO satisfies $(y \geq 0)$

$$
\begin{aligned}
m(y) & =\operatorname{meas}\left\{x \in(0,2 \pi):\left|f(x)-f_{(0,2 \pi)}\right|>y\right\} \\
& \leq 2 \pi \exp \left\{-\gamma y /\|f\|_{\text {BMO }}\right\},
\end{aligned}
$$

where $f_{(0,2 \pi)}$ is the average value of $f$ on $(0,2 \pi)$ and $\gamma$ is a positive numerical constant. Assuming $f_{(0,2 \pi)}=0,(9)$ implies

$$
\int_{0}^{2 \pi} \exp \left\{\frac{\gamma|f(x)|}{2\|f\|_{\mathrm{BMO}}}\right\} d x \leq C,
$$

where $C$ is another numerical constant. Putting $f=f_{r}$ and applying (7), we obtain (8).

\section{Radial growth.}

THEOREM 3. There is a numerical constant $\kappa$ such that, for every $u(z) \in$ $B, u(0)=0$,

$$
\limsup _{r \rightarrow 1^{-}} \frac{\left|u\left(r e^{\imath x}\right)\right|}{\sqrt{|\log (1-r)|} \log |\log (1-r)|} \leq \kappa\|u\|_{B}
$$

for almost all $x$.

PROOF. (8) implies that $(0<r<1)$

$$
\int_{0}^{1} \frac{d r}{(1-r) \log ^{2}(e / 1-r)} \int_{0}^{2 \pi} \exp \left\{\frac{c\left|u\left(r e^{i x}\right)\right|}{\|u\|_{B} \sqrt{\left|\log \left(1-r^{2}\right)\right|}}\right\} d x \leq C .
$$

Therefore, for almost all $x$,

$$
\int_{0}^{1} \frac{1}{(1-r) \log ^{2}(e / 1-r)} \exp \left\{\frac{c\left|u\left(r e^{\imath x}\right)\right|}{\|u\|_{B} \sqrt{\left|\log \left(1-r^{2}\right)\right|}}\right\} d r<\infty
$$

which implies that

$$
\lim _{r \rightarrow 1^{-}} \int_{r}^{(1+r) / 2}(1-\rho)^{-1} \log ^{-2}(e / 1-\rho) \exp \left\{\frac{c\left|u\left(r e^{i x}\right)\right|}{\|u\|_{B} \sqrt{\left|\log \left(1-\rho^{2}\right)\right|}}\right\} d \rho=0 .
$$

Putting $\mu(r, x)=\min \left\{\left|u\left(\rho e^{i x}\right)\right|: r \leq \rho \leq(1+r) / 2\right\}$, we get

$$
\lim _{r \rightarrow 1^{-}}\left(\frac{c \mu(r, x)}{\|u\|_{B} \sqrt{|\log (1-r)|}}-2 \log |\log (1-r)|\right)=-\infty .
$$

Thus, for almost all $x$, as $r \rightarrow 1^{-}, \mu(r, x)$ ultimately becomes

$$
<c_{1}\|u\|_{B} \sqrt{|\log (1-r)|} \log |\log (1-r)|,
$$

with some other constant $c_{1}$. On the other hand, (1) shows that the oscillation of $u(\rho, x)$ on the interval $r \leq \rho \leq(1+r) / 2$ does not exceed $\|u\|_{B}$, which proves (11) with $\kappa=c_{1}$. 


\section{REFERENCES}

1. J. G. Clunie and T. H. MacGregor, Radial growth of the derivative of univalent functions, Comment. Math. Helv. (to appear).

2. T. M. Flett, Some remarks on schlict functions and harmonic functions of uniformly bounded variation, Quart. J. Math Oxford Ser. 6 (1955), 59-72.

3. F. John and L. Nirenberg, On functions of bounded mean oscillation, Comm. Pure Appl. Math. 14 (1961), 415-426.

4. P. Koosis, Introduction to $H_{p}$ spaces, Cambridge Univ. Press, 1980.

5. Ch. Pommerenke, Univalent functions, Vandenhoeck and Ruprecht, Göttingen, 1975.

6. A. Zygmund, Trigonometric series, Cambridge, 1959.

Department of MATHEMAtics and Statistics, State UNiversity of NeW YORK, ALBANY, NEW YORK 12222 